\title{
CONDUTIVIDADE HIDRÁULICA SATURADA E NÃO SATURADA DE LATOSSOLO VERMELHO EUTROFÉRRICO TRATADO COM LODO DE ESGOTO(1)
}

\author{
G. M. C. BARBOSA ${ }^{(2)}$, J . TAVARES FILHO(3) \& I. C. B. FONSECA ${ }^{(3)}$
}

\begin{abstract}
RESUMO
E ste trabalho teve por objetivo avaliar os efeitos de diferentes doses de lodo de esgoto na condutividade hidráulica saturada e não saturada de um Latossolo Vermelho eutroférrico (Oxisol) durante dois anos consecutivos. $O$ experimento foi instalado no campo, com delineamento em blocos ao acaso com três repetições com os seguintes tratamentos: testemunha e adubação orgânica com lodo de esgoto em doses crescentes de 6, 12, 18, 24 e $36 \mathrm{Mg} \mathrm{ha}^{-1} \mathrm{em}$ base seca e complementação mineral. As medições de condutividade foram realizadas no campo com infiltrômetro de sucção controlada para os potenciais matriciais $\mathbf{0}$, $-1,-3$ e - $6 \mathrm{kPa}$. Nas condições em que foi realizado este experimento, concluiu-se que a dose de $12 \mathrm{Mg} \mathrm{ha}^{-1}$ de lodo de esgoto alterou a condutividade hidráulica nos potenciais 0 e $-1 \mathrm{kPa}$, respectivamente.
\end{abstract}

Termos de indexação: biossólido, potencial matricial, agregação do solo

\section{SUMMARY: SATURATED AND UNSATURATED HYDRAULIC CONDUCTI- VITY OF A SEWAGE SLUDGE TREATED "LATOSSOLO VERMELHO EUTROFÉRRICO" (OXISOL)}

This work aimed to eval uate the effects of sewage sludge application on the saturated and unsaturated hydraulic conductivity of a eutrophyc Red Latosol (Oxisol) during two consecutive years. This field experiment was conducted in a complete randomized block design with 3 replications and with the fol lowing treatments: control and increasing doses

(1) Parte da Tese de Mestrado, apresentada pelo primeiro autor para obtenção do título de Mestre em Agronomia pela Universidade Estadual de Londrina - UEL. Recebido para publicação em julho de 2002 e aprovado em janeiro de 2004.

(2) Doutoranda em Agronomia da Universidade Estadual de Londrina - UEL. Caixa Postal 6001, CEP $86051-990$ Londrina (PR). E-mail: obarbosa@sercomtel.com.br

(3) Professor do Departamento de Agronomia, UEL. E-mail: tavares@uel.br 
organic fertilization with sewage sludge $\left(6,12,18,24\right.$, and $36 \mathrm{Mg} \mathrm{ha}^{-1}$ on a dry basis) and mineral supplementation. The conductivity was measured in the fiedd with a controlled suction infiltrometer for thematric potentials of $0,-1,-3$ and $-6 \mathrm{kPa}$. Under theexperi mental conditions of the present experiment, it was conduded that a sewagesludgedose of $12 \mathrm{Mg} \mathrm{ha}^{-1}$ al tered the hydraulic conductivity at the potentials of 0 and $-1 \mathrm{kPa}$, respectively.

Index terms: biosolids, matric potentials, soil aggregation.

\section{INTRODUÇÃO}

O lodo de esgoto nos países desenvolvidos tem sido utilizado, predominantemente, em terras produtivas reservadas para agricultura e pecuária, bem como depositado em aterros sanitários (Luduvice, 2000). Essa reciclagem transforma o lodo de esgoto em um importante insumo agrícola, que fornece matéria orgânica e nutrientes ao solo e vantagens indiretas ao homem e ao ambiente, não só por reduzir os efeitos adversos à saúde causados pela incineração, mas também por diminuir a dependência de fertilizantes químicos (Andreoli \& Pegorini, 2000).

Uma característica importante do lodo de esgoto é a quantidade e a qualidade da matéria orgânica, que pode variar enormemente de acordo com sua procedência e época do ano (Melo \& Marques, 2000). Ela desempenha um complexo papel na dinâmica dos sol os, influindo em suas características químicas, físico-químicas, biológicas e físicas.

A aplicação do lodo de esgoto no solo aumenta o seu teor de matéria orgânica (Bataglia et al., 1983; Outwater, 1994) e, como conseqüência, tem-se uma melhora na capacidade de armazenamento e de infiltração da água no solo, bem como na conduti vidade hidráulica próxima à saturação (Morel et al., 1978; Chang et al., 1983; Wei et al., 1985; Marciano et al., 2001; Barbosa et al., 2002), pelo aumento da porosidade total (Kladivko., 1979; J orge et al., 1991; Lue-Hing et al., 1992; Wisniewski et al., 1996), aeração e formação de agregados (Carvalho \& Barral, 1981; Bernardes, 1982; Tsutya, 2000; Marciano et al., 2001) e diminuição da densidade (Bernardes,1982; Bonnet, 1995). Segundo Gupta et al. (1977), a condutividade hidráulica diminui conforme o aumento da dose de lodo nas parcelas de sol os não saturados.
A condutividade hidráulica comporta-se de maneira diferente para cada tipo de solo. Assim, este trabalho teve por objetivo avaliar os efeitos de diferentes doses de lodo de esgoto na condutividade hidráulica de um Latossolo Vermel ho eutroférrico.

\section{MATERIAL E MÉTODOS}

O trabalho foi realizado em um Latossolo Vermel ho eutroférrico, com teor de argila variando de 760 a $820 \mathrm{~g} \mathrm{~kg}^{-1}$ entre $0-0,40 \mathrm{~m}$, localizado no município de L ondrina (PR), coordenadas geográficas $23^{\circ} 23^{\prime}$ de latitude $S$ e 51 $11^{\prime}$ de longitude $W$, altitude média 566 m e clima, segundo classificação de Köppen, do tipo Cfa - subtropical úmido.

O lodo utilizado (Quadro 1) foi digerido anaerobiamente, produzido em estação do tipo Reator Anaeróbio de Lodo F luidizado - RALF, etratado com cal (dolomítica) na concentração de $50 \%$ do peso de lodo seco, com umidade variando de 50 a $75 \%$.

O solo do experimento recebeu calagem e, posteriormente, foi gradeado. A pós estas operações, foi realizada a apl icação de lodo manual mentea cada seis meses e, em seguida, efetuou-se nova gradagem superficial $(0-0,10 \mathrm{~m})$ para incorporação. As dosagens totais de lodo de esgoto foram aplicadas durante dois anos, anterior ao plantio da safra de verão e, neste período, foram plantadas as culturas de aveia e mil ho nas safras de 1997, 1997/98 e 1998/ 99 (verão/inverno). Após o período de dois anos, cessada a aplicação dol odo de esgoto, fez-se o plantio de milho safrinha (1999) e deu-se início à coleta de dados no campo.

$\mathrm{O}$ delineamento experimental foi em blocos. A área total do experimento foi de 0,5 ha (três blocos, com seis tratamentos), totalizando 18 parcelas. Os

Quadro 1. Características químicas de uma amostra de lodo anaeróbio (RALF) utilizado na região de Londrina (PR) (adaptado de Andreoli, 1999)

\begin{tabular}{ccccccccc}
\hline $\mathbf{p H}\left(\mathbf{H}_{\mathbf{2}} \mathbf{O}\right)$ & $\mathbf{P}_{\mathbf{2}} \mathbf{O}_{5}$ total & $\mathbf{K}_{\mathbf{2}} \mathbf{O}$ & $\mathbf{C a}^{2+}$ & $\mathbf{M g}^{2+}$ & $\mathbf{N}$ total & $\mathbf{M . O}$. total & $\mathbf{C}$ total & $\mathbf{C} / \mathbf{N}$ \\
\hline & 9,5 & 3,4 & 8,3 & 3,0 & 22,2 & 362,0 & 201,0 & $9 / 1$ \\
\hline
\end{tabular}


tratamentos receberam doses anuais de lodo de esgoto divididos da seguinte forma: testemunha (calagem de $1 \mathrm{t} \mathrm{ha}^{-1}$ ); $6 \mathrm{t} \mathrm{ha}^{-1}$ de lodo calado; $12 \mathrm{t} \mathrm{ha}^{-1}$ de lodo calado; $18 \mathrm{t} \mathrm{ha}^{-1}$ de lodo calado; $24 \mathrm{t} \mathrm{ha}^{-1}$ de lodo calado, e $36 \mathrm{t} \mathrm{ha}^{-1}$ de lodo calado. Todos os tratamentos que utilizaram o lodo de esgoto receberam complementação mineral no verão (300 kg ha-1), da formulação (08-18-16).

Após a colheita (manual) da safra de verão (milho), foi plantado o milho safrinha sem qual quer tipo de adubação. Em março de 1999, durante o desenvolvimento da cultura do milho safrinha, foi determinada a condutividade hidráulica por meio de infiltrômetro de sucção controlada (Ankeny et al., 1991), para as tensões 0, 1, 3 e 6 kPa na superfície. Foi aplicada uma cobertura no solo com areia de diâmetro $\leq 100 \mu \mathrm{m}$, para obter o contato adequado entre o tecido de náil on (infiltrômetro de sucção) e o solo. Nos locais onde foi determinada a condutividade hidráulica, coletaram-se amostras de solo para análise da estabilidade de agregados em água (método deY oder), visando caracterizar a estrutura do solo (Quadro 2).
A análise estatística dos dados da condutividade hidráulica foi feita segundo esquema fatorial em blocos $6 \times 4$ (seis tratamentos $\times$ quatro potenciais) com três repetições. Os dados foram submetidos à análise de variância e teste de comparações de médias de Tukey a $5 \%$ e análise de regressão dos dados da condutividade deacordo coma dose (H offman \& Vieira, 1983) para cada potencial estudado.

\section{RESULTADOS E DISCUSÃO}

Comparando os diferentes potenciais dentro de cada tratamento, percebeu-se que somente o tratamento $12 \mathrm{Mg} \mathrm{ha}^{-1}$ mostrou diferença significativa de condutividade: maiores valores, 431,0 e $404,5 \mathrm{~mm} \mathrm{~h}^{-1}$, nos potenciais 0 e -1 kPa (Quadro 3). Para estes potenciais em que foram detectadas as diferenças entre as doses, buscou-se realizar uma análise polinomial, porém nenhum ajuste até o 3o grau foi adequado para estes dois potenciais. Estes resultados estão de acordo com os de Marciano

Quadro 2. Médias da estabilidade de agregados do Latossolo Vermelho eutroférrico utilizado no estudo

\begin{tabular}{|c|c|c|c|c|c|c|}
\hline \multirow{2}{*}{$\begin{array}{l}\text { Tratamento - dose } \\
\text { de lodo calado }\end{array}$} & \multicolumn{2}{|r|}{ DMP } & \multicolumn{2}{|r|}{ DMG } & \multicolumn{2}{|r|}{ IEA } \\
\hline & Média & Desvio-padrão & Média & Desvio-padrão & Média & Desvio-padrão \\
\hline & \multicolumn{4}{|c|}{$-\mathrm{mm}$} & \multicolumn{2}{|c|}{$\%$} \\
\hline Testemunha & 3,61 & 0,41 & 1,25 & 0,17 & 80,57 & 2,66 \\
\hline $6 \mathrm{Mg} \mathrm{ha}^{-1}$ & 4,46 & 0,23 & 1,64 & 0,07 & 83,88 & 1,29 \\
\hline $12 \mathrm{Mg} \mathrm{ha}^{-1}$ & 5,10 & 0,69 & 2,37 & 0,89 & 87,90 & 4,42 \\
\hline $18 \mathrm{Mg}$ ha-$^{-1}$ & 4,63 & 1,22 & 1,95 & 0,49 & 89,01 & 6,25 \\
\hline $24 \mathrm{Mg} \mathrm{ha}^{-1}$ & 4,10 & 0,58 & 1,68 & 0,08 & 87,64 & 5,52 \\
\hline $36 \mathrm{Mg} \mathrm{ha}^{-1}$ & 4,78 & 0,25 & 1,93 & 0,27 & 86,69 & 2,15 \\
\hline
\end{tabular}

DMP = Diâmetro médio ponderado; DMG = Diâmetro médio geométrico, e IEA = Índice de estabilidade de agregados.

Quadro 3. Efeito residual do lodo de esgoto na condutividade hidráulica do Latossolo Vermelho eutroférrico utilizado no estudo

\begin{tabular}{|c|c|c|c|c|}
\hline \multirow{2}{*}{$\begin{array}{l}\text { Tratamento - dose } \\
\text { de lodo calado }\end{array}$} & \multicolumn{4}{|c|}{ Potencial matricial } \\
\hline & $0 \mathrm{kPa}$ & $-1 \mathrm{kPa}$ & $-3 \mathrm{kPa}$ & $-6 \mathrm{kPa}$ \\
\hline Testemunha & $60,5 \mathrm{Ba}^{(1)}$ & $104,0 \mathrm{Ba}$ & $37,5 \mathrm{Aa}$ & $33,0 \mathrm{Aa}$ \\
\hline $6 \mathrm{Mg} \mathrm{ha}^{-1}$ & $48,5 \mathrm{Ba}$ & $33,5 \mathrm{Ba}$ & $14,5 \mathrm{Aa}$ & $5,0 \mathrm{Aa}$ \\
\hline $12 \mathrm{Mg} \mathrm{ha}^{-1}$ & $431,0 \mathrm{Aa}$ & $404,5 \mathrm{Aa}$ & $23,5 \mathrm{Ab}$ & $10,0 \mathrm{Ab}$ \\
\hline $18 \mathrm{Mg}$ ha- 1 & $90,0 \mathrm{Ba}$ & $56,5 \mathrm{Ba}$ & $10,5 \mathrm{Aa}$ & 4,0 Aa \\
\hline $24 \mathrm{Mg} \mathrm{ha}^{-1}$ & $100,5 \mathrm{Ba}$ & $80,0 \mathrm{Ba}$ & $21,0 \mathrm{Aa}$ & $13,0 \mathrm{Aa}$ \\
\hline $36 \mathrm{Mg} \mathrm{ha}^{-1}$ & $61,5 \mathrm{Ba}$ & $82,5 \mathrm{Ba}$ & $11,0 \mathrm{Aa}$ & $4,5 \mathrm{Aa}$ \\
\hline C.V. (\%) & \multicolumn{4}{|c|}{44,98} \\
\hline
\end{tabular}

\footnotetext{
(1) Médias seguidas de mesma letra maiúscula, na coluna, e minúscula, na linha, não diferem entre si pelo teste de Tukey a 5 \%.
} 
(1999) que constatou mai ores variações no conteúdo de água nos potenciais matriciais próximos à saturação er redução na condutividade hidráulica do solo nos outros potenciais. Por outro lado, J orge et al. (1991) observaram que em oito tratamentos analisados, com e sem lodo de esgoto, não houve efeito sobre as propriedades de infiltração.

Ao comparar os tratamentos dentro de cada potencial, observou-se nos potenciais 0 e- $1 \mathrm{kPa}$, que otratamento $12 \mathrm{Mg} \mathrm{ha}^{-1}$ diferiu dos demais. Nestes potenciais em que foram detectadas diferenças entre as doses, buscou-se realizar uma anál ise polinomial, porém nenhum ajuste até 30 grau foi adequado. Estes resultados estão de acordo com os obtidos por Morel et al. (1978), Chang et al. (1983), Wei et al. (1985) e Marciano (2001), cujos resultados de condutividade hidráulica demonstraram não haver efeito significativo dos tratamentos para os potenciais próximos à saturação e sua redução à medida que o solo se afasta da saturação. Nos potenciais -3 e $-6 \mathrm{kPa}$, não houve diferença significativa da condutividade hidráulica entre os tratamentos, confirmando dados obtidos por Gupta et al. (1977).

Verificou-se maior condutividade hidráulica para os potenciais 0 e $-1 \mathrm{kPa}$, quando utilizado o lodo de esgoto, menor para os potenciais - 3 e - $6 \mathrm{kPa}$ para as mesmas parcelas. Esses resultados decondutividade hidráulica obtidos podem estar refletindo o fato de existir mel hor agregação do solo (maior DMP, maior DMG e mais de $87 \%$ de IEA) (Quadro 2), quando utilizada a dose de $12 \mathrm{Mg} \mathrm{ha}^{-1}$. Portanto, uma macroporosidade com poros maiores de $150 \mu \mathrm{m}$ parece existir nessesolo, influenciando positivamente a infiltração da água para os potenciais 0 e-1 kPa.

SegundoEpstein (1975), oaumento da estabilidade de agregados do solo influi na infiltração de água, além do fato de a matéria orgânica e os cátions presentes $\left(\mathrm{Ca}^{2+}\right.$ e $\mathrm{Al}^{3+}$ dentre outros) no lodo de esgoto influenciarem a agregação de partículas do sol o e determinarem o aumento do seu vol ume (M elo \& Marques, 2000). Em solo submetido a outros potenciais (-3 e -6 kPa), a movimentação de água, em uma porosidade menor que $150 \mu \mathrm{m}$, fica limitada, dificultando, assim, a condutividade da água (LueHing et al., 1992; Logan \& Harrison, 1995; Barbosa et al., 2002).

Valeainda ressaltar que, na avaliação em campo com infiltrômetro de sucção controlada para as parcelas que receberam mais de $12 \mathrm{Mg} \mathrm{ha}^{-1}$ de lodo de esgoto, foi observada repelência da água na superfície, dificultando sua infiltração. Este fenômeno foi comprovado por Constantini et al. (1995) e Marciano et al. (2001) e, segundo os primeiros autores, esta repelência diminuiu a taxa de infiltração inicial na superfície do solo, fato que contribuiu, provavelmente, para a redução da condutividade hidráulica nos tratamentos com 18, 24 e $36 \mathrm{Mg} \mathrm{ha}^{-1}$.

\section{CONCLUSÃO}

1. Verificou-se que a dose de $12 \mathrm{Mg} \mathrm{ha}^{-1}$ de lodo de esgoto aumentou a condutividade hidráulica nos potenciais 0 e $-1 \mathrm{kPa}$, respectivamente.

\section{LITE RATURA CITADA}

ANDREOLI, C.V. Uso e manejo do lodo de esgoto na agricultura e sua influência em características ambientais no agrossistema. Curitiba, Universidade Estadual do Paraná, 1999. 278p. (Tese de Doutorado)

ANDREOLI, C.V.\& PEGORINI, E.S. Gestão pública do uso agrícola do lodo de esgoto. In: I mpacto ambiental do uso do lodo de esgoto. J aguariúna, EMBRAPA Meio Ambiente, 2000. p.281-312.

ANKENY, M.D.; AHMED, M.; KASPAR, T.C. \& HORTON, R. Simple field method for determining unsaturated hydraulic conductivity. Soil Sci. Soc. Am. J ., 55:470-476, 1991.

BARBOSA, G.M.C.; TAVARES FILHO, J . \& FONSECA, I.C.B. Avaliações das propriedades físicas em $L$ atossolo Vermel ho eutroférrico tratado com lodo de esgoto por dois anos consecutivos. Sanare, 17:94-101, 2002.

BATAGLIA, O.C.; BERTON, R.S.; CAMARGO, A.O. \& VALADARES, J .M.A.S. Resíduos orgânicos como fontes de nitrogênio para capim-braquiária. R. Bras. Ci. Solo, 7:277-284, 1983.

BERNARDES, L.F. Efeitos da aplicação do lodo de esgoto nas propriedades físicas do solo. J aboticabal, U niversidade Estadual de São Paulo, 1982. 50p. (Tese de Mestrado)

BONNET, B.R.P. Diagnóstico da situação e posição preliminar de sistema de monitoragem dos impactos ambientais causados pelo uso agrícola do lodo de esgoto no Paraná. Curitiba, Universidade Federal do Paraná, 1995. Não publicado.

CARVALHO, P.C. \& BARRAL, M. F. Aplicação do lodo de esgoto como fertilizante. Fertilizantes, 3:3-5, 1981.

CHANG. A.C.; PAGE, A.L. \& VARNEKE, J .E. Soil conditioning effects of municipal sludge compost. J. Environ. Eng., 109:574-583, 1983.

CONSTANTINI, A.; LOCH, R.J .; GLANVILLE, S.F . \& ORANGE, D.N. Evaluation of the potencial to dispose of sewage sludge. I. Soil hydraulic and overland flow properties of pinus plantations in Queensland. Aust. J . Soil Res., 33:10411052, 1995.

EPSTEIN, E. Effect of sewage sludge on some soil physical properties. J Environ. Qual., 5:422-426, 1975.

GUPTA, S.C.; DOWDY, R.H. \& LARSON, W.E. Hidraulic and thermal properties of a sandy soil as influenced by incorporation of sewage sludge. Soil Sci Soc. Am. J ., 41:601605, 1977.

HOFFMANN, R. \& VIEIRA, S. Análise de regressão: Uma condução à econometria. 2.ed. São Paulo, HUCITEC, 1983. 
J ORGE, J .A.; CAMARGO, O.A. \& VALADARES, J.M.A.S. Condições físicas de um $L$ atossolo Vermel ho-E scuro quatro anos após aplicação de lodo de esgoto e calcário. R. Bras. Ci. Solo, 15:237-240, 1991.

KLADIVKO, E.J \& NELSON, D.W. Changers in soil properties from application of anaerobic sludge. J . Water Poll., 51:325332, 1979.

LOGAN, T.J . \& HARRISON, B.J . Physical characteristics of alkaline stabilized sewage sludge ( $\mathrm{N}$ - Viro Soil) and their effects on soil physical properties. J . Environ. Qual., 24:153-164, 1995.

LUDUVICE, M. Experiência da Companhia de Saneamento do Distrito Federal na reciclagem agrícola de biossólido. In: Impacto ambiental do uso do lodo de esgoto. J aguariúna: EMBRAPA Meio Ambiente, 2000. p.153-162.

LUE-HING, C.; ZENZ, D.R. \& KUCHENRITHER. Municipal sewage sludge management: processing, utilization and disporal. Water Qual. Manag., 4:455-500, 1992.

MARCIANO, C.R. Incorporação de resíduos urbanos e as propriedades físico-hídricas de um Latossolo VermelhoAmarelo. Piracicaba, Escola Superior de Agricultura "Luiz de Queiroz", 1999. 93p. (Tese de Doutorado)

MARCIANO, C.R.; MORAES, S.O.; OLIVEIRA, F.C. \& MATTIAZZO, M.E. Efeito do lodo de esgoto e do composto de lixo urbano sobre a condutividade hidráulica de um Latossolo Amarelo saturado e não saturado. R. Bras. Ci. Solo, 25:1-9, 2001.
MAZURAK, A.P.; CHESNIN, L. \& TIARKS, E. Detachment of soil aggregation by simulated from heavily manured soils in Eastern Nebraska. Soil Sci. Soc. Am. Proc., 30:732736,1975 .

MELO, W.J . \& MARQUES, M.O. Potencial do lodo de esgoto como fonte de nutriente para as plantas. In: Impacto ambiental do uso do lodo deesgoto. J aguariúna, EMBRAPA Meio Ambiente, 2000. p.109-141.

MOREL, J .L.; GUCKERT, A. \& SEGODO, M. Effects de l'épandage des boues résiduaires urbaines sur l'état physique du sol. Bull. Ensaia, 20:13-19, 1978.

OUTWATER, A.B. Reuse of sludge and minor wastewater residuals. [S.1.], Lewis Publishers, 1994. 179p.

TSUTYA, M.T. Alternativas de disposição final de biossólidos gerados em estações de tratamento de esgotos. In: I mpacto ambiental do uso do lodo de esgoto. J aguariúna, EMBRAPA Meio Ambiente, 2000. p.69-105.

WEI, Q.F.; LOWERY, B. \& PETERSON, A.E. Effect of sludge aplication on physical properties of a silty clay loam soil. J . Environ. Qual., 14:178-180, 1985.

WISNIEWSKI, C. NETO, J .A. \& PEREIRA, A.M. Uso do lodo de esgoto da ETE - Belém na recuperação de áreas degradadas por mineração de calcário. Sanare, 5:76-86, 1996. 
G.M.C. BARBOSA et al. 